\title{
Clinical utility of rosuvastatin and other statins for cardiovascular risk reduction among the elderly
}

This article was published in the following Dove Press journal:

Clinical Interventions in Aging

22 December 2010

Number of times this article has been viewed

\section{Sydney B Long \\ Michael J Blaha \\ Roger S Blumenthal \\ Erin D Michos}

Johns Hopkins Ciccarone Center for the Prevention of Heart Disease, Baltimore, MD, USA
Correspondence: Erin D Michos

Division of Cardiology, Johns

Hopkins School of Medicine,

Carnegie 568, 600 NWolfe Street,

Baltimore, MD 21287, USA

Tel +l 4l0 5026813

Fax + I 4I0 $502023 \mid$

Email edonnell@jhmi.edu

\begin{abstract}
Age is one of the strongest predictors of cardiovascular disease (CVD) risk. Treatment with statins can significantly reduce CVD events and mortality in both primary and secondary prevention. Yet despite the high CVD risk among the elderly, there is underutilization of statins in this population (ie, the treatment-risk paradox). Few studies have investigated the use of statins in the elderly, particularly for primary prevention and, as a result, guidelines for treating the elderly are limited. This is likely due to: uncertainties of risk assessment in older individuals where the predictive value of individual risk factors is decreased; the need to balance the benefits of primary prevention with the risks of polypharmacy, health care costs, and adverse medication effects in a population with decreased life expectancy; the complexity of treating patients with many other comorbidities; and increasingly difficult social and economic concerns. As life expectancy increases and the total elderly population grows, these issues become increasingly important. JUPITER (Justification for the Use of statins in Prevention: an Intervention Trial Evaluating Rosuvastatin) is the largest primary prevention statin trial to date and enrolled a substantial number of elderly adults. Among the 5695 JUPITER participants $\geq 70$ years of age, the absolute CVD risk reduction associated with rosuvastatin was actually greater than for younger participants. The implications of this JUPITER subanalysis and the broader role of statins among older adults is the subject of this review.
\end{abstract}

Keywords: JUPITER, rosuvastatin, elderly, risk

\section{Introduction}

Cardiovascular disease (CVD) is the leading cause of death for both men and women in the US. ${ }^{1}$ In addition to causing significant mortality and morbidity, CVD is responsible for a very large portion of medical spending. CVD accounts for the most annual hospital discharges of the 10 leading diagnostic groups (6.2 million in 2006), and the direct costs of CVD are estimated to be $\$ 324.1$ billion in the US in $2010 .{ }^{1}$ The benefits for the appropriate use of evidence-based preventive therapies are widespread, and include reductions in CVD events, disability, and health care expenditure (ie, costly hospitalizations and institutionalizations), as well as improvement in quality of life. Thus, strategies for optimizing the prevention and treatment of CVD in the elderly are of paramount importance from both the individual and societal perspectives. ${ }^{2}$

It has long been acknowledged that increasing age is one of the strongest risk factors for the development of CVD. The prevalence of CVD increases with age, from $15 \%$ in men and $9 \%$ in women aged $20-39$ years to $79 \%$ in men and $85 \%$ in women aged $80+$ years. $^{1}$ As such, higher age categories are the primary determinants of increased risk in global risk assessment tools, such as the Framingham Risk Score (FRS), 
which take into account age, gender, total and high-density lipoprotein cholesterol (HDL-C), smoking, and blood pressure. ${ }^{3}$ The National Cholesterol Education Program (NCEP) Adult Treatment Panel (ATP)-III guidelines use the FRS to set lipid treatment guidelines based on the FRS-determined 10-year global risk for coronary heart disease (CHD). ${ }^{3}$ However, the FRS was designed for those $<80$ years of age and becomes less accurate in the elderly population. Furthermore, limitations of the FRS scoring system for underestimating CVD risk in other populations have been acknowledged as well. ${ }^{4-6}$

Age is perhaps the strongest prognostic factor in clinical medicine, and age commonly dictates treatment plans because of marked biologic differences between age groups. The division of people into distinct age groups allows for more focused study and development of specific clinical guidelines. In most developed countries, the age at which people are considered "elderly" has been conventionally set at 65 years, which is often associated with retirement age or the age when one begins to receive pension benefits in that country. The physiologic changes, as a result of aging, occur along a continuum rather than in discrete age groups, and life expectancies continually change as medical advances are made. This has resulted in an evolving concept of the "elderly". Some have suggested further dividing the elderly into three groups, ie, 65-74 years, 75-85 years, and $\geq 85$ years, for better understanding of the different biologic processes that occur with age. ${ }^{7}$

Elderly adults with CVD, or at increased risk for CVD, are an increasingly challenging subset of the population to treat, because they are more likely to have comorbid conditions, atypical presentations, and unfavorable outcomes compared with their younger counterparts. ${ }^{8}$ Evidence-based medicine for CVD prevention in subjects over the age of 80 years is limited and frequently extrapolated from studies of younger individuals. ${ }^{9}$

Multiple observational and clinical trials have confirmed that increased low-density lipoprotein cholesterol (LDL-C) levels are associated with greater risk for new-onset CHD among people without a history of CHD and increased recurrent coronary events in people with established CHD. The development of statins, or HMG-CoA reductase inhibitors, has greatly added to the arsenal of medical therapy for the treatment and prevention of CVD, and has contributed to the reduction of CVD-related mortalities. The NCEP ATP-III recommends that, for high-risk patients, the treatment LDL-C goal is $<100 \mathrm{mg} / \mathrm{dL}$, but that an LDL-C goal of $<70 \mathrm{mg} / \mathrm{dL}$ is a therapeutic option for those at very high risk or those with baseline LDL-C of $<100 \mathrm{mg} / \mathrm{dL}^{3}$
Unfortunately, treatment gaps are well documented; a 2003 national survey found that only $57 \%$ of high-risk individuals with CHD or risk equivalent achieved the recommended LDL-C goal of $<100 \mathrm{mg} / \mathrm{dL}$ and only $18 \%$ achieved the goal of $<70 \mathrm{mg} / \mathrm{dL}$. $^{10}$

However, few studies have investigated the use of statins in the elderly, particularly for primary prevention and, as a result, guidelines for treating the elderly are limited. JUPITER (Justification for the Use of statins in Prevention: an Intervention Trial Evaluating Rosuvastatin) is the largest primary prevention statin trial to date, and enrolled a substantial number of elderly adults. ${ }^{11}$ The implications of JUPITER, the role of rosuvastatin for CVD risk reduction in the elderly, and the broader role of statins among older adults are discussed here. For this review, the search terms "rosuvastatin", "statins", "elderly", "older adults", and "cardiovascular disease" were entered into PubMed, and selected clinical trials and observational studies (based on size, importance to the field, and direct relevance to this review topic) were chosen by the authors to present in this review.

\section{Pharmacology, mode of action, and pharmacokinetics of rosuvastatin}

There are several ways in which statins improve the lipid profile. Statins work by inhibiting the actions of HMG-CoA reductase, an enzyme responsible for the conversion of $\mathrm{HMG}$ $\mathrm{CoA}$ to mevalonate in the rate-limiting step of intracellular cholesterol biosynthesis. This leads to decreased LDL-C and very-low-density lipoprotein (VLDL-C) synthesis. ${ }^{12}$ With decreased intracellular stores of cholesterol, cells upregulate the expression of LDL receptors on the surface of the liver and increase the uptake of LDL-C and VLDL-C from the circulation. Most of the statins also modestly raise HDL-C. The exact mechanisms are unclear, but could possibly involve decreasing the fractional catabolic rate of apolipoprotein A-I, increasing apolipoprotein A-I production, and/or inhibiting cholesteryl ester transfer protein activity.

Statin use often results in clinical benefits greater than what might be expected from changes in lipid levels alone. This is due to possible pleiotropic effects of statins, which include improving endothelial function, stabilizing atherosclerotic plaques, decreasing oxidative stress and inflammation, and inhibiting the thrombogenic response. ${ }^{13}$

Rosuvastatin is a highly effective statin medication that is the most potent of its class in reducing LDL-C and raising HDL-C levels. In STELLAR (Statin Therapies for Elevated Lipid Levels compared Across doses to Rosuvastatin) ${ }^{14}$ and the MERCURY (Measuring Effective Reductions in 
Cholesterol Using Rosuvastatin therapY) I-II trials, ${ }^{15,16}$ patients in the rosuvastatin groups were able to achieve greater reduction in LDL-C than patients on other statins. Because multiple clinical trial results suggest that greater lowering of LDL-C and lower LDL-C level treatment goals are beneficial in populations with existing CVD or at risk of it, ${ }^{17-20}$ the role of more potent statins, such as rosuvastatin, might increase. On the other hand, the second most potent statin, atorvastatin, will likely be available as a generic medication in 2012.

The increased LDL-C-lowering potency of rosuvastatin over other statins can be attributed to its unique chemical properties that give it the highest affinity for HMG-CoA reductase. ${ }^{12}$ A fluorinated phenyl group and a polar methylsulfonamide group on rosuvastatin provides multiple sites of activity against the enzyme. Rosuvastatin is also hydrophilic and does not require metabolic conversion to a water-soluble molecule for elimination, which reduces the number of drug-drug interactions that other more hydrophobic statins encounter. In healthy volunteers, $90 \%$ of orally administered [14C]-radiolabeled rosuvastatin was recovered in feces in its unmetabolized form. Lastly, both rosuvastatin and atorvastatin have enhanced binding enthalpies for $\mathrm{HMG}-\mathrm{CoA}$ reductase, due to formation of a hydrogen bond with the enzyme that causes greater inhibition of the enzyme.

Rosuvastatin has an oral bioavailability of $20 \%$, similar to that of atorvastatin, pravastatin, and fluvastatin, with peak plasma concentrations reached 3-5 hours after oral administration and an elimination half-life for rosuvastatin of approximately 19 hours. ${ }^{12}$ Importantly, there were no significant differences in plasma rosuvastatin concentrations or reductions in LDL-C levels relative to age or gender of the subjects. Rosuvastatin is predominantly cleared by the hepatic route, with only minimal renal clearance $(28 \%)$, and the dose does not need to be adjusted in those with mild to moderate renal impairment. ${ }^{21}$

\section{Statins for secondary prevention in older adults}

People with established CHD are at high risk for recurrent events. The use of LDL-C-lowering therapy has been shown to reduce the incidence of recurrent coronary events in people with established CHD in multiple secondary prevention trials, even before the development of statins. ${ }^{22}$ Since the introduction of statins, secondary prevention trials with statins, such as 4S (the Scandinavian Simvastatin Survival Study), ${ }^{23}$ the CARE (Cholesterol And Recurrent Events) study, ${ }^{24}$ the LIPID (Long-term Intervention with
Pravastatin in Ischemic Disease) study, ${ }^{25}$ and the HPS (Heart Protection Study) ${ }^{26}$ have shown that participants with CHD and/or diabetes treated with simvastatin or pravastatin had decreased incidences of major coronary events, revascularization, coronary mortality, strokes, and total mortality.

HPS, which enrolled 9839 participants aged $<65$ years, 4891 participants aged 65-70 years, and 5806 participants aged $>70$ years, showed that the proportional reduction in event rates was similar across age categories. ${ }^{26}$ The study demonstrated that statins had substantial benefit in older age as well as in middle age for secondary prevention. In the PROVE IT-TIMI 22 (Pravastatin or Atorvastatin Evaluation and Infection Therapy - Thrombolysis in Myocardial Infarction 22) study, ${ }^{19}$ intensive lipid-lowering statin therapy was found to be superior to standard statin therapy in protecting against death or major cardiovascular events in patients who suffered a recent acute coronary syndrome and also found that these patients benefited from further lowering of LDL-C. In this trial, there was no significant interaction for treatment effect heterogeneity across the age subgroups ( $<65$ years or $\geq 65$ years).

While the results for older patients $>65$ years were analyzed in many clinical trials as part of age-specific subgroup analysis, two secondary prevention studies, ie, PROSPER (PrOspective Study of Pravastatin in the Elderly at Risk) and SAGE (Study Assessing Goals in the Elderly) enrolled only older adults. PROSPER ${ }^{27}$ was conducted to evaluate the role of pravastatin $40 \mathrm{mg}$ /day compared with placebo in reducing coronary and cerebral events in elderly patients aged 70-82 years (mean baseline age 75 years) with pre-existing vascular disease or who were at high risk of vascular disease and stroke; most would say this represents a combination of primary and secondary prevention.

PROSPER randomized 5804 participants within this older age range and having total plasma cholesterol 155-350 mg/dL and triglyceride levels $<200 \mathrm{mg} / \mathrm{dL}$ to treatment with pravastatin or placebo. After a mean follow up of 3.2 years, it was found that participants in the pravastatin group had 34\% lower LDL-C levels compared with placebo and had a statistically significant $15 \%$ relative risk reduction in the incidence of coronary death, nonfatal myocardial infarction, and fatal or nonfatal stroke. However, there were more new cancer diagnoses in the pravastatin group compared with placebo. Subsequent large meta-analyses of statin trials did not confirm an increase risk of cancer associated with statin treatment. ${ }^{28}$

In $\mathrm{SAGE},{ }^{29}$ researchers compared the effects of intensive versus moderate statin therapy in 893 older patients aged 65-85 years (mean age 72 years) with one or more 
episodes of myocardial ischemia during 48-hour ambulatory electrocardiography. The participants were randomized to atorvastatin $80 \mathrm{mg} /$ day or pravastatin $40 \mathrm{mg} /$ day. While there was no difference between the two groups in the primary endpoint of duration of ischemia on ambulatory monitoring at 12 months, intensive atorvastatin therapy was associated with greater reductions in cholesterol, a trend toward fewer major acute cardiovascular events (hazard ratio [HR] 0.71; 95\% CI $0.46-1.09 ; P=0.114)$, and statistically significant reduction in all-cause death (HR $0.33 ; 95 \%$ CI $0.13-0.83 ; P=0.014$ ). The authors concluded that these findings, together with the increased cardiovascular risk in the elderly population, suggest that intensive statin therapy should be considered in elderly patients with CHD.

Clinical trials frequently have multiple exclusion criteria and, thus, elderly patients with many comorbidities (such as renal failure or insufficiency, etc) may be excluded from participation. The efficacy of statin use for secondary prevention among the very elderly has been examined in a real-world clinical registry setting. Gransbo et al studied 14,907 patients over the age of 80 years who were admitted with an acute myocardial infarction in the Register of Information and Knowledge About Swedish Heart Intensive Care Admissions (RIKS-HIA) and followed for a median of 296 days (maximum of five years). ${ }^{30}$ Among the very elderly patients receiving statin therapy at discharge, there was a $45 \%$ lower risk of allcause mortality (relative risk [RR] 0.55, 95\% CI 0.51-0.59). Mortality reduction with statin use was similar when deaths within 14 days or within 365 days of the index event were excluded from analysis. This real-world data confirms that, even among the very elderly, a CVD risk reduction benefit is seen even within a short time period, and supports close consideration of statins for high-risk patients, regardless of age.

\section{Statins for primary prevention in older adults}

Multiple clinical trials have suggested a benefit of statins in primary prevention, although these trials generally enrolled a younger population. WOSCOPS (West of Scotland Coronary Prevention Study) studied men (mean baseline age 55 years, with an upper age limit of only 64 years) with moderate hypercholesterolemia on initial screening but no history of myocardial infarctions or cardiac revascularization. ${ }^{31}$ Subgroup analyses showed that the relative reductions in CHD risk attributable to pravastatin therapy was not affected by age ( $<55$ versus $\geq 55$ years).

In the MEGA (Management of Elevated Cholesterol in the Primary Prevention Group of Adult Japanese) study, ${ }^{32} 8214$ patients, including men aged $40-70$ years and postmenopausal women aged up to 80 years (mean baseline age 58 years) with hypercholesterolemia and no history of CHD or stroke were randomized to diet alone or diet plus pravastatin groups. At the mean follow-up of 5.3 years, assignment to pravastatin therapy conferred a $33 \% \mathrm{RR}$ reduction in CHD. Subgroup analysis of the risk reduction of CHD with pravastatin did not show significant interactions in any subgroup, including age $<60$ and $\geq 60$ years.

The AFCAPS/TexCAPS (Air Force/Texas Coronary Atherosclerosis Prevention Study $)^{33}$ randomized 6605 participants aged 45-73 years with average total cholesterol and LDL-C and below-average HDL-C levels to lovastatin or placebo. The baseline mean age of the participants was 58 years, with $21 \%$ older than 65 years. After a follow-up of 5.2 years, participants in the lovastatin group were found to have LDL-C levels reduced by $25 \%$, HDL-C levels increased by $6 \%$, and a significant $37 \%$ RR reduction in the rate of first acute major coronary event, defined as the composite endpoint of fatal or nonfatal myocardial infarction, unstable angina, or sudden cardiac death. Further subgroup analysis based on gender-stratified median age (which was $>57$ years in men and 62 years in women) versus $\leq$ median age did not show any significant differences in benefit with lovastatin in the two age groups. The authors concluded that there was no reason to believe older people did not benefit as much as younger ones from lovastatin therapy.

ASCOT (the Anglo-Scandinavian Cardiac Outcomes Trial) randomized 19,342 hypertensive patients aged 40-79 years (mean baseline age 63 years) with three other cardiovascular risk factors (ie, a higher risk primary prevention cohort) to one of two antihypertensive regimens. In ASCOT-LLA (the Lipid Lowering Arm of the study), ${ }^{34}$ 10,305 of the hypertensive patients not deemed to be hypercholesterolemic (with total nonfasting cholesterol $<250 \mathrm{mg} / \mathrm{dL}$ ) were randomized to additional treatment with atorvastatin $10 \mathrm{mg}$ or placebo. The trial was planned for follow-up in five years, but was prematurely stopped after a median follow-up of 3.3 years due to a significant reduction in the primary endpoint of nonfatal myocardial infarction and fatal CHD for atorvastatin compared with placebo (HR 0.64, 95\% CI 0.50-0.83, $P=0.0005$ ). The effect of atorvastatin on the primary endpoint was not significantly different in any prespecified subgroup, including those specified as younger ( $\leq 60$ years) or older ( $>60$ years). Of note, in PROSPER, ${ }^{27}$ there was no reduction in strokes seen for patients $>70$ years with pravastatin. However, a subanalysis of $\mathrm{ASCOT}^{34}$ did find that stroke prevention with atorvastatin was similar among the 2416 patients $>70$ years 
compared with those $\leq 70$ (31\% versus $24 \%$ relative stroke reduction).

\section{JUPITER trial of statins in primary prevention}

As described above, previous primary prevention trials required hyperlipidemia or low HDL-C at enrollment. JUPITER, ${ }^{11}$ the largest primary prevention statin trial to date, was a multicenter, randomized, double-blind, placebo-controlled trial enrolling men $\geq 50$ years and women $\geq 60$ years (median baseline age 66 years) who had no history of CVD, no hypercholesterolemia (LDL-C level $<130 \mathrm{mg} / \mathrm{dL}$ ), a high-sensitivity C-reactive protein (hsCRP) level $\geq 2.0 \mathrm{mg} / \mathrm{L}$, and a triglyceride level $<500 \mathrm{mg} / \mathrm{dL}$ at the initial screening visit. After an initial four-week run in phase to identify individuals with good compliance, the eligible participants were randomized to receive either rosuvastatin $20 \mathrm{mg}$ daily, or matching placebo. Of the 89,890 people screened for enrollment, 17,802 were eligible to participate (of which the majority were excluded for not meeting the LDL-C or hsCRP criteria). Compared with previous trials, JUPITER enrolled an older population with no upper age limit.

JUPITER was terminated after a median follow-up of 1.9 years due to a statistically significant reduction in the primary endpoint of myocardial infarction, stroke, arterial revascularization, hospitalization for unstable angina, or death from cardiovascular causes in the rosuvastatin group compared with placebo (HR 0.56, 95\% CI 0.46-0.69, $P<0.00001$ ). The rosuvastatin group had a 50\% lower median LDL-C level and a $37 \%$ lower median hsCRP level than the placebo group at 12 months that persisted throughout the study period. Based on Kaplan-Meier estimates, the number needed to treat (NNT) with rosuvastatin for two years to prevent the occurrence of one primary endpoint is 95 . Extrapolated out to five years, the NNT for the primary endpoint is 25 , and including venous thromboembolic events, the NNT is only $18 .^{35}$

In subgroup analyses, the authors found no evidence for heterogeneity in the results for any subgroup, with similar relative hazard reductions in the rosuvastatin group noted in subgroups according to age ( $\leq 65$ and $>65$ years), gender, race or ethnic group, region of origin, FRS, and status with regard to traditional risk factors. ${ }^{11}$ For subjects with elevated hsCRP levels but no other major risk factor other than the increased age at enrollment required for eligibility, the benefit of rosuvastatin was similar to that for higher risk subjects (HR 0.63, 95\% CI 0.44-0.92, $P=0.01$ ).

A secondary analysis of JUPITER examined the 5695 participants $\geq 70$ years at baseline to evaluate the efficacy and safety of rosuvastatin in older adults. ${ }^{36}$ The authors chose the cutpoint of age 70 years because of the lack of data on the use of statins for primary prevention in that age group. This elderly group comprised $32 \%$ of the total number of trial participants, but accounted for $49 \%$ of the 393 confirmed primary cardiovascular endpoints. This elderly group was also noted to have a different profile of other cardiovascular risk factors when compared with the younger participants, with higher percentages of women and individuals with hypertension, and lower percentages of obesity and cigarette smoking in the elderly group.

The reduction in lipid levels and hsCRP levels seen in the rosuvastatin group was similar in the elderly group and the younger group. The median LDL-C levels in the rosuvastatin group $(54 \mathrm{mg} / \mathrm{dL}$ and $55 \mathrm{mg} / \mathrm{dL}$ in older and younger participants, respectively) were half those in each age group that received placebo. The median hsCRP levels (2.3 and $2.2 \mathrm{mg} / \mathrm{L}$ in older and younger participants, respectively) were nearly $36 \%$ lower in the rosuvastatin versus placebo group, separately in each age group.

Rates of the primary endpoint among JUPITER participants $\geq 70$ years were 1.22 and 1.99 per 100 person-years of follow-up in the rosuvastatin and placebo groups, respectively (HR 0.61, 95\% CI 0.46-0.82). This RR reduction is somewhat more modest than compared with younger individuals aged 50-69 years (HR 0.51, 95\% CI 0.38-0.69), which is consistent with a meta-analysis of observation studies reporting that a $1 \mathrm{mmol} / \mathrm{L}$ (39 mg/dL) lower total cholesterol level was associated with a $56 \%$ reduction (CI 52\%-58\%) in the hazard of death from ischemic heart disease at age 40-49 years, but a 17\% reduction (CI 15\%-19\%) at age 70-89 years. ${ }^{37}$ Yet, in JUPITER, there was no statistically significant interaction between age and treatment effect for any outcome ( $P>0.10$ for each).

Furthermore, when combined with the fact that the elderly group had higher rates of cardiovascular events, the absolute reduction in the incidence of the primary endpoint associated with rosuvastatin was actually $48 \%$ greater ( 0.77 versus 0.52 events per 100 person-years) in the elderly group compared with the younger group. ${ }^{36}$ The estimated NNT of elderly persons for four years to prevent one primary endpoint was 24 (CI 15-57) compared with 36 (CI 23-77) in the younger group. There were similar hazard reductions in both elderly men and women, and no significant heterogeneity was observed across subgroups among the elderly participants.

Older participants in the JUPITER study did have higher rates of adverse events (including muscle weakness, newly diagnosed cancers, renal disorder, gastrointestinal or hepatic 
disorder, and bleeding) compared with younger adults. However, these adverse effects were not more common in older patients treated with rosuvastatin compared with placebo $(P>0.10$ for all adverse events), indicating the safety of rosuvastatin treatment in this age group. However, it should be remembered that the mean follow-up for this trial was only about two years.

Of note, several limitations of the overall JUPITER study design have been acknowledged, ${ }^{38}$ including the possibility that the premature termination of the trial might have exaggerated the benefits. ${ }^{39}$ However, an independent review by the US Food and Drug Administration of the JUPITER trial concluded that any potential overestimate of the RR reduction due to early stopping would be minimal and not change the overall study implications. ${ }^{40}$ Also, only those with elevated hsCRP $\geq 2 \mathrm{mg} / \mathrm{L}$ were enrolled, so it still remains unclear whether all older adults, regardless of hsCRP level, might benefit from statin therapy by virtue of increased baseline risk.

Using data from the National Health and Nutrition Examination Survey 1999-2004, we estimated that 6.5 million additional adults, not currently eligible for statin therapy by the NCEP ATP-III criteria, could potentially be candidates for initiating statins according to the JUPITER criteria. ${ }^{41}$ Thus, using the JUPITER eligibility strategy, an estimated 260,000 CVD events could be prevented in the US at five years. ${ }^{41}$ The fourth Adult Treatment Panel is currently convening to weigh the evidence from the more recent statin clinical trials that have taken place since 2004, and updated lipid guidelines are anticipated to be released in 2011.

\section{Rosuvastatin for prevention in special populations}

On the other hand, two important studies, ie, AURORA (A study evaluating the Use of Rosuvastatin in patients requiring Ongoing Renal dialysis: an Assessment of survival and cardiovascular events) and CORONA (Controlled Rosuvastatin Multinational Trial in Heart Failure), evaluating the efficacy of rosuvastatin in specific populations found that while rosuvastatin did reduce the levels of LDL-C and hsCRP, there was no difference in the rate of primary endpoints in the rosuvastatin groups compared with placebo.

Several studies have shown that patients undergoing maintenance hemodialysis have an increased risk of CVD, and observational studies have suggested that statin therapy can have survival benefits in patients undergoing hemodialysis. AURORA ${ }^{42}$ was a prospective trial that randomized 2776 patients, aged $50-80$ years (mean baseline age
64 years), who were undergoing maintenance hemodialysis for advanced renal failure to receive rosuvastatin $10 \mathrm{mg}$ daily or placebo. After three months of treatment, patients randomized to the rosuvastatin group had LDL-C levels that were $43 \%$ lower than their baseline level as compared with only a $2 \%$ reduction from baseline in the placebo group. The median hsCRP level decreased by $12 \%$ in the rosuvastatin group (by $0.65 \mathrm{mg} / \mathrm{L}$, versus an increase of $0.21 \mathrm{mg} / \mathrm{L}$ in the placebo group, $P<0.001)$.

After a median follow-up of 3.8 years, the primary endpoint of death from cardiovascular causes, nonfatal myocardial infarction, or nonfatal stroke occurred in 396 patients in the rosuvastatin group ( 0.2 events per 100 patient-years) versus 408 patients in the placebo group ( 9.5 events per 100 patientyears), with no significant effect of treatment (HR 0.96, 95\% CI $0.84-1.11, P=0.59)$. The lack of an effect of rosuvastatin therapy on the primary endpoint was consistent in all the prespecified subgroups, including patients younger than 65 years and those aged $\geq 65$ years.

These findings suggest that the CVD process in patients undergoing hemodialysis differs from that in other patient populations. In the general population, a majority of cardiovascular events are coronary events such as myocardial infarctions. In the hemodialysis population, however, only approximately $25 \%$ of cardiovascular events are myocardial infarctions. ${ }^{43}$ Rather, heart failure, sudden cardiac death, and arrhythmias predominate in this population. Therefore, the anti-inflammatory and lipid-lowering effects of statins may not benefit a population in which myocardial infarctions do not predominate.

CORONA $^{44}$ investigated the use of rosuvastatin in older patients with systolic heart failure, a population also generally excluded from statin trials. The study randomized 5011 patients aged at least 60 years (mean baseline age 73 years, with $41 \%$ at least 75 years) with New York Heart Association Class II, III, or IV ischemic systolic heart failure to rosuvastatin $10 \mathrm{mg}$ daily or placebo. After a median follow-up of 32.8 months, patients in the rosuvastatin group had decreased levels of LDL-C (45\% difference between groups, $P<0.001)$ and hsCRP $(37 \%$ difference between groups) compared with placebo. However, there was no statistically significant decrease in the primary outcome, that included death from cardiovascular causes, nonfatal myocardial infarction, or nonfatal stroke (HR 0.92, 95\% CI $0.83-1.02, P=0.12)$. On the other hand, there was a statistically significant reduction in the number of hospitalizations for cardiovascular causes in the rosuvastatin group compared with the placebo group ( $P<0.001)$, which was a secondary endpoint for this trial. 
The authors of the study had hypothesized that statin use would stabilize coronary plaques and reduce myocardial ischemia and infarction, and thereby decrease the rate of sudden death in patients with ischemic heart failure, a population in which half of the sudden deaths are caused by plaque rupture. While it is unclear why treatment with rosuvastatin did not decrease the rate of the primary outcome, the authors suggested that alternative mechanisms of death, such as pump failure rather than atherosclerotic causes, effects of other drugs the patients were on, and the need for a longer follow-up period to see beneficial effects of treatment as possible explanations.

A post hoc analysis from CORONA did suggest a significant interaction by hsCRP status $(P$ interaction $=0.026)$ with rosuvastatin benefitting those with hsCRP $\geq 2 \mathrm{mg} / \mathrm{L}$ but not those with low hsCRP. ${ }^{45}$ Furthermore, an economic analysis of the overall CORONA cohort, including both the primary outcome and the secondary outcome of hospitalizations, did find that the overall reduction in CVD events with rosuvastatin partially offset the costs of rosuvastatin treatment by $44 \%$, thus finding rosuvastatin treatment to be a cost-effective treatment for older patients with systolic heart failure. ${ }^{46}$

\section{Risk-treatment paradox in the elderly}

Elderly patients have the highest CVD risk, and yet there is an underutilization of statin therapy in this population (ie, the treatment-risk paradox). ${ }^{47,48}$ Using registry data of nearly 400,000 patients $>66$ years in Ontario who had a prior history of CVD or diabetes, the adjusted likelihood of statin prescription was $6.4 \%$ lower for each one year in age and each $1 \%$ increase in predicted three-year mortality risk. ${ }^{49}$ Gnavi et al looked at prescribing practices of statins for residents of Torino, Italy, aged 30-85 years in 2001-2002 with a hospital discharge diagnosis of ischemic heart disease by comparing with the regional database for drug prescriptions. The authors found that, among those $>74$ years of age, statin prescription rates were $40 \%$ lower than for younger individuals. ${ }^{50}$

A more recent US survey conducted in 2005-2006 reviewed medical records from 4964 primary care visits for patients aged 55-80 years with cerebrovascular disease, ischemic heart disease, peripheral arterial disease, aortic aneurysms, diabetes mellitus, or any two risk factors (hyperlipidemia, hypertension, or smoking). The authors found that only $38 \%$ were prescribed a statin, a far lower amount than would be anticipated from their comorbidities. ${ }^{51}$ This problem of undertreatment in the elderly is not exclusive to statin therapy, because many proven therapies, including thrombolytic therapy ${ }^{52}$ and beta-blockers, ${ }^{53}$ have been reported to be underutilized in the elderly presenting with acute myocardial infarction.

\section{Resistance to statin use in the elderly}

As described above, in randomized controlled trials of both secondary and primary prevention, supported by registry data, statins have been shown to reduce CHD events and in some cases all-cause mortality. This finding applies across nearly all available studies, with the possible exception of highly specialized populations, such as systolic heart failure and end-stage renal disease requiring hemodialysis.

While the evidence for statins for secondary prevention in the elderly is more established (despite their underutilization), there are fewer primary prevention studies dedicated to enrolling large numbers of elderly patients, and thus data to support guidelines in this age group are limited. This is possibly due in part to the increasing uncertainties of risk assessment in older individuals as the predictive value of risk factors declines. Other issues to take into consideration include the increased need to balance the benefits of primary prevention with the risks of polypharmacy, health care costs, and adverse medication effects in a population with decreased life expectancy, increased number of coexisting diseases, and more difficult social and economic situations. As the population of the elderly continue to increase with medical advances, these issues are becoming more important.

Of note, a small but statistically significant increased risk of incident diabetes has been reported with statin therapy compared with placebo in a recent large meta-analysis of statin clinical trials (odds ratio 1.09, 95\% CI 1.02-1.17), a risk that was highest in trials with older participants such as those in PROSPER and JUPITER ${ }^{54}$ This translated to one new case of diabetes per 1000 person-years of treatment, but extended over four years, nine vascular events would be prevented for each new case of diabetes. Thus, it is generally thought that the risk of incident diabetes is low when compared with the significant reduction in CVD events. However, some authors have suggested that the risk/benefit ratio of treatment may not be as favorable in subjects with a propensity to develop diabetes, such as the elderly, and warrants further study. ${ }^{55}$

Low to moderate doses of statins do appear to be well tolerated in the elderly. The safety of statin therapy in the elderly can be enhanced by avoiding concomitant use of $\mathrm{P} 450$ inhibitors, chronic immunosuppressive therapy (cyclosporine), or fibrates (gemfibrozil), and by limiting concomitant use of niacin and alcohol intake (less than two drinks daily). 
Obviously, laboratory screening for renal, liver, and thyroid function should be performed before initiation of statin therapy. Consider starting statin therapy at lower doses and titrating slowly (toxicity is dose-related). If there is severe medical illness, major surgery, or major trauma, the discontinuation of statin therapy until recovery can be considered.

\section{Conclusion}

In summary, there is a need for further study regarding the role of statin therapy for primary prevention in the elderly, especially for those without overt hyperlipidemia who do not currently meet the NCEP-ATP III guidelines for initiation of statin therapy and among the elderly with well controlled blood pressures. ${ }^{56}$ Furthermore, the risk of new-onset diabetes with statin therapy should be evaluated more closely in the elderly population.

However, in the absence of medical contraindications, at this time the authors feel there is sufficient evidence to support the recommendation for use of statin treatment in most elderly patients with risk profiles similar to those enrolled in prior primary and secondary prevention trials.

\section{Disclosure}

None of the authors have any disclosures or conflicts of interest related to this topic. EDM is supported by the PJ Schafer Foundation Preventive Cardiovascular Research Fund and a Career Development Award from the American College of Cardiology.

\section{References}

1. Heart disease and stroke statistics - 2010 update. Available at: http://www.americanheart.org/presenter.jhtml?identifier=3018163. Accessed 2010 Nov 22.

2. Gopal A, Budoff MJ. Coronary calcium scanning in geriatric cardiology. Am J Geriatr Cardiol. 2007;16:369-375.

3. Executive Summary of The Third Report of The National Cholesterol Education Program (NCEP) Expert Panel on Detection, Evaluation, And Treatment of High Blood Cholesterol In Adults (Adult Treatment Panel III). JAMA. 2001;285:2486-2497.

4. Akosah KO, Schaper A, Cogbill C, et al. Preventing myocardial infarction in the young adult in the first place: How do the National Cholesterol Education Panel III guidelines perform? J Am Coll Cardiol. 2003; 41:1475-1479.

5. Michos ED, Nasir K, Braunstein JB, et al. Framingham risk equation underestimates subclinical atherosclerosis risk in asymptomatic women. Atherosclerosis. 2006;184:201-206.

6. DeMazumder D, Hasan RK, Blumenthal RS, Michos ED, Jones S. Should statin therapy be allocated on the basis of global risk or on the basis of randomized trial evidence? Am J Cardiol. 2010;106:905-909.

7. Alexander KP, Newby LK, Cannon CP, et al. Acute coronary care in the elderly, Part I: Non-ST-segment-elevation acute coronary syndromes: A scientific statement for healthcare professionals from the American Heart Association Council on Clinical Cardiology, in collaboration with the Society of Geriatric Cardiology. Circulation. 2007;115:2549-2569.

8. Keller NM, Feit F. Coronary artery disease in the geriatric population. Prog Cardiovasc Dis. 1996;38:407-418.
9. Hanon O. Coronary artery disease in patients 80 years and older. Rev Prat. 2008;58:156-170. French.

10. Davidson MH, Maki KC, Pearson TA, et al. Results of the National Cholesterol Education (NCEP) Program Evaluation ProjecT Utilizing Novel E-Technology (NEPTUNE) II Survey and implications for treatment under the recent NCEP Writing Group recommendations. Am J Cardiol. 2005;96:556-563.

11. Ridker PM, Danielson E, Fonseca FA, et al; for the JUPITER study group. Rosuvastatin to prevent vascular events in men and women with elevated C-reactive protein. N Engl J Med. 2008;359:2195-2207.

12. Kapur NK. Rosuvastatin: A highly potent statin for the prevention and management of coronary artery disease. Expert Rev Cardiovasc Ther. 2007:5:161-175.

13. Yildirir A, Muderrisoglu H. Non-lipid effects of statins: Emerging new indications. Curr Vasc Pharmacol. 2004;2:309-318.

14. Jones PH, Davidson MH, Stein EA, et al; STELLAR Study Group. Comparison of the efficacy and safety of rosuvastatin versus atorvastatin, simvastatin, and pravastatin across doses (STELLAR trial). Am J Cardiol. 2003;92:152-160.

15. Schuster H, Barter PJ, Stender S, et al; Effective Reductions in Cholesterol Using Rosuvastatin Therapy I study group. Effects of switching statins on achievement of lipid goals: Measuring Effective Reductions in Cholesterol Using Rosuvastatin Therapy (MERCURY I). Am Heart J. 2004; 147:705-713.

16. Ballantyne CM, Bertolami M, Hernandez Garcia HR, et al. Achieving LDL cholesterol, non-HDL cholesterol, and apolipoprotein B target levels in high-risk patient: Measuring Effective Reductions in Cholesterol Using Rosuvastatin therapy (MERCURY II). Am Heart J. 2006; 151:975.e1-979.e1.

17. Ridker PM, Danielson E, Fonseca FA, et al. Reduction in C-reactive protein and LDL cholesterol and cardiovascular event rates after initiation of rosuvastatin: A prospective study of the JUPITER trial. Lancet. 2009;373:1175-1182.

18. LaRosa JC, Grundy SM, Waters DD, et al; for the Treating to New Targets (TNT) Investigators. Intensive lipid lowering with atorvastatin in patients with stable coronary disease. $N$ Engl J Med. 2005; 352:1425-1435.

19. Cannon CP, Braunwald E, McCabe $\mathrm{CH}$, et al; for the Pravastatin or Atorvastatin Evaluation and Infection Therapy-Thrombolysis in Myocardial Infarction 22 Investigators (PROVE-IT TIMI 22). Intensive versus moderate lipid lowering with statins after acute coronary syndromes. N Engl J Med. 2004;350:1495-1504.

20. Pederson TR, Cater NB, Faergeman O, et al. Comparison of atorvastatin $80 \mathrm{mg} /$ day vs simvastatin $20-40 \mathrm{mg}$ /day on frequency of cardiovascular events late (five years) after acute myocardial infarction (from the Incremental Decrease in End Points through Aggressive Lipid Lowering [IDEAL] trial). Am J Cardiol. 2010;106:354-359.

21. Tzeng TB, Schneck DW, Birmingham BK, et al. Population pharmacokinetics of rosuvastatin: Implications of renal impairment, race, and dyslipidaemia. Curr Med Res Opin. 2008;24:2575-2585.

22. Clofibrate and niacin in coronary heart disease. JAMA. 1975; 231:360-381.

23. The Scandinavian Simvastatin Survival Study Group. Randomized trial of cholesterol lowering in 4444 patients with coronary heart disease: The Scandinavian Simvastatin Survival Study (4S). Lancet. 1994; 344:1383-1389.

24. Sacks FM, Pfeffer MA, Moye LA, et al. The effect of pravastatin on coronary events after myocardial infarction in patients with average cholesterol levels. Cholesterol and Recurrent Events Trial Investigators. N Engl J Med. 1996;335:1001-1009.

25. The Long-Term Intervention with Pravastatin in Ischaemic Disease (LIPID) Study Group. Prevention of cardiovascular events and death with pravastatin in patients with coronary heart disease and a broad range of initial cholesterol levels. N Engl J Med. 1998;339:1349-1357.

26. Heart Protection Study Collaborative Group. MRC/BHF Heart Protection Study of cholesterol lowering with simvastatin in 20536 high-risk individuals a randomized placebo controlled trial. Lancet. 2002;360:7-22. 
27. Sheperd J, Blauw GJ, Murphy MB, et al; for the PROSPER study group. Pravastatin in elderly individuals at risk of vascular disease (PROSPER): A randomized controlled trial. Lancet. 2002;360:1623-1630.

28. Brugts JJ, Yetgin T, Hoeks SE, et al. The benefits of statins in people without established cardiovascular disease but with cardiovascular risk factors: Meta-analysis of randomized controlled trials. BMJ. 2009; 338:b2376.

29. Deedwania P, Stone PH, Bairey Merz CN, et al. Effect of intensive vs moderate lipid-lowering therapy on myocardial ischemia in older patients with coronary heart disease: Results of the Study Assessing Goals in the Elderly (SAGE). Circulation. 2007;116:700-707.

30. Gransbo K, Melander O, Wallentin L, et al. Cardiovascular and cancer mortality in very elderly post-myocardial infarction patients receiving statin treatment. J Am Coll Cardiol. 2010;55:1362-1369.

31. Shepherd J, Cobbe SM, Ford I, et al; for the West of Scotland Coronary Prevention Study Group (WOSCOPS). Prevention of coronary heart disease with pravastatin in men with hypercholesterolemia. $N$ Engl J Med. 1995;333:1301-1307.

32. Nakamura $\mathrm{H}$, Arakawa $\mathrm{K}$, Itakura $\mathrm{H}$, et al; for the MEGA group. Primary prevention of cardiovascular disease with pravastatin in Japan (MEGA Study): A prospective randomized controlled trial. Lancet. 2006;368:1155-1163.

33. Downs JR, Clearfield M, Weis S, et al. Primary prevention of acute coronary events with lovastatin in men and women with average cholesterol levels: Results of (AFCAPS/TexCAPS) Air Force/Texas Coronary Atherosclerosis Prevention Study. JAMA. 1998;279:1615-1622.

34. Sever PS, Dahlof B, Poulter NR, et al; for the ASCOT investigators. Prevention of coronary and stroke events with atorvastatin in hypertensive patients who have average or lower-than average cholesterol concentrations, in the Anglo-Scandinavian Cardiac Outcomes Trial - Lipid Lowering Arm (ASCOT-LLA): A multicenter randomized controlled trial. Lancet. 2003;361:1149-1158.

35. Glynn RJ, Danielson E, Fonseca FA, et al. A randomized trial of rosuvastatin in the prevention of venous thromboembolism. $N$ Engl J Med. 2009;360:1851-1861.

36. Glynn RJ, Koenig W, Nordestgaard BG, Sheperd J, Ridker PM. Rosuvastatin for primary prevention in older persons with elevated C-reactive protein and low to average low density lipoprotein cholesterol levels: Exploratory analysis of a randomized trial. Ann Intern Med 2010;152:488-496.

37. Lewington S, Whitlock G, Clarke R, et al. Prospective Studies Collaboration. Blood cholesterol and vascular mortality by age, sex, and blood pressure: A meta-analysis of individual data from 61 prospective studies with 55,000 vascular deaths. Lancet. 2007;370:1829-1839.

38. Narla V, Blaha MJ, Blumenthal RS, Michos ED. The JUPITER and AURORA clinical trials for rosuvastatin in special primary prevention populations: Perspectives, outcomes, and consequences. Vasc Health Risk Manag. 2009;5:1033-1042.

39. Bassler D, Briel M, Montori VM, et al. Stopping randomized trials early for benefit and estimation of treatment effects: Systematic review and meta-regression analysis. JAMA. 2010;303:1180-1187.

40. Ridker PM, Glynn RJ. The JUPITER trial: Responding to the critics. Am J Cardiol. 2010;106:1351-1356.
41. Michos ED, Blumenthal RS. Prevalence of low low-density lipoprotein cholesterol with elevated high sensitivity C-reactive protein in the US. J Am Coll Cardiol. 2009;53:931-935.

42. Fellström BC, Jardine AG, Schmieder RE, et al. Rosuvastatin and cardiovascular events in patients undergoing hemodialysis. $N$ Engl J Med. 2009;360:1395-1407.

43. Foley RN, Murray AM, Li S, et al. Chronic kidney disease and the risk of cardiovascular disease, renal replacement, and death in the United States Medicare population, 1998 to 1999. J Am Soc Nephrol. 2005; 16:489-495.

44. Kjekshus J, Apetrei E, Barrios V, et al; for the CORONA Group. Rosuvastatin in older patients with systolic heart failure. $N$ Engl $J$ Med. 2007;357:2248-2261.

45. McMurray JJ, Kjekshus J, Gullestad L, et al; for the CORONA Study Group. Effects of statin therapy according to plasma high-sensitivity C-reactive protein concentration in the Controlled Rosuvastatin Multinational Trial in Heart Failure (CORONA): A retrospective analysis. Circulation. 2009;120:2188-2196.

46. Lorgelly PK, Briggs AH, Wedel H, et al; for the CORONA Study Group. An economic evaluation of rosuvastatin treatment in systolic heart failure: Evidence from the CORONA trial. Eur J Heart Fail. 2010; 12:66-74.

47. Lemaitre RN, Furberg CD, Newman AB, et al. Time trends in the use of cholesterol-lowering agents in older adults: The Cardiovascular Health Study. Arch Intern Med. 1998;158:1761-1768.

48. Whincup PH, Emberson JR, Lennon L, Walker M, Papacosta O, Thomson A. Low prevalence of lipid lowering drug use in older men with established coronary heart disease. Heart. 2002;88:25-29.

49. Ko DT, Mamdani M, Alter DA. Lipid-lowering therapy with statins in high risk elderly patients: The treatment-risk paradox. JAMA. 2004; 291:1864-1870

50. Gnavi R, Migliardi A, Demaria M, Petrelli A, Caprioglio A, Costa G. Statins prescribing for the secondary prevention of ischaemic heart disease in Torino, Italy. A case of ageism and social inequalities. Eur J Public Health. 2007;17:492-496.

51. Niska R, Han B. Statins for secondary cardiovascular disease prevention for older primary care patients. J Natl Med Assoc. 2009; 101:705-710.

52. Krumholz HM, Murillo JE, Chen J, et al. Thrombolytic therapy for eligible elderly patients with acute myocardial infarction. JAMA. 1997; 277:1683-1688.

53. Rochon PA, Anderson GM, Tu JV, et al. Use of beta-blocker therapy in older patients after acute myocardial infarction in Ontario. CMAJ 1999;161:1403-1408.

54. Sattar N, Preiss D, Murray HM, et al. Statins and the risk of incident diabetes: A collaborative meta-analysis of randomized statin trials. Lancet. 2010;375:735-742.

55. Athyros VG, Tziomalos K, Karagiannis A, Mikhailidis DP. Lipid-lowering agents and new onset diabetes mellitus. Expert Opin Pharmacother. 2010;11:1965-1970

56. Robinson JG, Bakris G, Torner J, Stone NJ, Wallace R. Is it time for a cardiovascular primary prevention trial in the elderly? Stroke. 2007; $38: 441-450$
Clinical Interventions in Aging

\section{Publish your work in this journal}

Clinical Interventions in Aging is an international, peer-reviewed journa focusing on evidence-based reports on the value or lack thereof of treatments intended to prevent or delay the onset of maladaptive correlates of aging in human beings. This journal is indexed on PubMed Central, MedLine, the American Chemical Society's 'Chemical Abstracts Ser-

\section{Dovepress}

vice' (CAS), Scopus and the Elsevier Bibliographic databases. The manuscript management system is completely online and includes a very quick and fair peer-review system, which is all easy to use. Visit http://www.dovepress.com/testimonials.php to read real quotes from published authors. 